\title{
GREEN'S FUNCTION, RESOLVENT, PARSEVAL EQUALITY OF DIFFERENTIAL OPERATOR WITH BLOCK-TRIANGULAR MATRIX COEFFICIENTS
}

\author{
A. M. Khol Kin And F. S. Rofe-Beketov
}

Abstract. Green's function for the Sturm-Liouville operator with a block-triangular matrix potential growing at infinity is constructed. For this function, a series expansion is obtained and the Parseval equality is proved.

Mathematics subject classification (2010): 34K11, 47A10. equality.

Keywords and phrases: Spectrum, block-triangular matrix coefficients, Green's function, Parseval

\section{REFERENCES}

[1] M. S. Agranovich and V. A. Marchenko, The Inverse Problem of Scattering Theory, Kharkov State University, Kharkov, 1960 (Russian) (Engl. transl.: N.Y.-London, Gordon \& Breach, 1963).

[2] E. I. BondarenKo And F. S. Rofe-BeKetov, Phase equivalent matrix potential, Electromagnetic waves and electronic systems, 5, No. 3, (2000), 6-24 (Russian) (Engl. transl.: Telecommun. And Radio Eng. 56 (8 and 9), (2001), 4-29).

[3] E. I. Bondarenko And F. S. Rofe-Beketov, Inverse Scattering Problem on the semiaxis for a system with a triangular matrix potential, Math. Physics, Analysis, and Geometry, 10, No. 3, (2003), 412-424 (Russian).

[4] A. ERemenko, A. Gabrielov, Spectral loci of Sturm-Liouville operators with polynomial potentials, to appear

[5] A. M. Kholkin And F. S. Rofe-Beketov, Sturm type oscillation theorems for equations with block - triangular matrix coefficients, Methods Funct. Anal. and Topology, 18, No. 2, (2012), 176188.

[6] A. M. Kholkin And F. S. Rofe-Beketov, On spectrum of differential operators with blocktriangular matrix coefficients, Math. Physics, Analysis, and Geometry, 10, 1 (2014), 44-63.

[7] M. Lesch AND M. M. Malamud, On the deficiency indices and selfadjointness of symmetric Hamiltonian systems, J. of Diff. Equations, vol. 189 (2003), 556-615.

[8] V. A. MARChENKo AND F. S. ROFE-BeKetov, Expansion in eigenfunctions of non-self-adjoint singular differential operators, Dokl. Akad. Nauk. SSSR 120, No. 5, (1958), 963-966 (Russian).

[9] V. A. Marchenko, Spectral Theory of Sturm-Liouville Operators, Naukova Dumka, Kiev, 1972. (Russian).

[10] V. A. Marchenko, Sturm-Liouville Operators and its applications, Naukova Dumka, Kiev, 1977. (Russian) (Engl. transl.: Birkhauser Verlag, Basel, 1986; revised edition AMS Chelsea Publishing, Providence R. I., 2011)

[11] F. S. Rofe-BeKeTOV, Expansion in eigenfunctions of infinite systems of differential equations in the non-self-adjoint and self-adjoint cases, Mat. Sb., 51, No. 3, (1960), 293-342 (Russian).

[12] F. S. Rofe-BeKetov AND E. KH. Khristov, Asymptotic and analytic questions connected with scattering by a highly singular potential, Collection of Proceeding of Fiz. Tekh. Inst. Niz. Temp. Akad. Nauk Ukrain. SSR. Mathematics, Physics and Functional Analysis Series, Khar'kov, No. 2, (1971), 122-168 (Russian). 
[13] F. S. Rofe-Beketov And A. M. Kholkin, Spectral Analysis of Differential Operators. Interplay between spectral and oscillatory properties, With Foreword by V. A. Marchenko, World Scientific, New Jersey, London, Singapore, Beijing, Shanghai, Hong Kong, Taipei, Chennai, 2005.

[14] E. Ch. Titchmarsh, Eigenfunction expansions associated with second - order differential equations, Vol. 2, Clarendon Press, Oxford, 1958. (Russian transl.: Vol. 2, Izdat. Inostran. Lit., Moscow, 1961). 